\title{
THE IMPORTANCE OF TIME SERIES DATA FILTERING FOR PREDiCTING THE Direction OF STOCK MARKeT MOVEMENT USING NEURAL NETWORKS
}

\author{
Ive Botunac, Ante Panjkota \& Maja Matetic
}
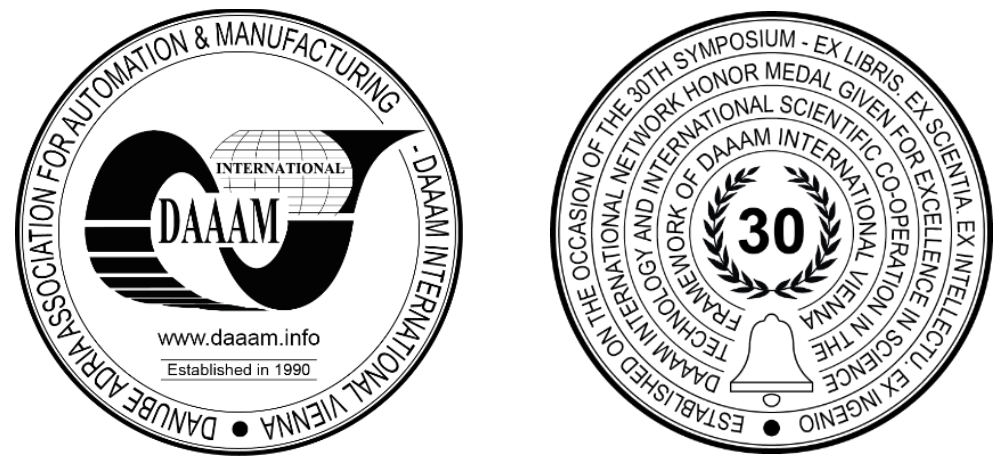

This Publication has to be referred as: Botunac, I[ve]; Panjkota, A[nte] \& Matetic, M[aja] (2019). The Importance of Time Series Data Filtering for Predicting the Direction of Stock Market Movement Using Neural Networks, Proceedings of the 30th DAAAM International Symposium, pp.0886-0891, B. Katalinic (Ed.), Published by DAAAM International, ISBN 978-3-902734-22-8, ISSN 1726-9679, Vienna, Austria

DOI: $10.2507 / 30$ th.daaam.proceedings.123

\begin{abstract}
Predicting future trends in the stock market from time-series data is a challenging task due to its high non-linear nature caused by the complexity involved in the trading process. This paper emphasizes the importance of time-series data filtering when neural network models are used for stock market direction forecasting. Performances of three different neural network models are compared on raw data, processed data with simple moving average, and data filtered with discrete wavelet transformation. Applying wavelet transformation on input financial data as a processing step shows better results than the use of raw financial data or simple moving average. Also, among tested neural network models, the better results are obtained by using long short-term neural network then by using other neural network models.
\end{abstract}

Keywords: stock market prediction; machine learning; neural network; wavelet transformation

\section{Introduction}

As a cause of its complex nature, the stock market is often marked by volatile and non-linear movements, which makes it difficult to predict future trends. Stock price and trend is often influenced by some critical and key factors such as political situations, businesses, certain economic activities and other unexpected events that may occur [1]. Similarly, movements can also be influenced by various automated systems that are increasingly trading in these markets. All these factors need to be taken into account when predicting future prices or direction of the movement of shares because it is of crucial importance to investors as it may reduce the risk of making bad decisions.

When predicting the movement of financial time series, different types of technical, fundamental and statistical approaches are used. For example, we can enumerate a simple moving average, linear regression, support vectors machine and artificial neural networks [2], [13]. In accordance to the results of some of the research [3], [4], by combining machine learning methods, it's visible that stock market trends can be predicted to a certain degree of accuracy which is often over $70 \%$. The wavelet transformation technique has been successfully applied in many areas encountering engineering 
problems as well as stock market forecasts due to its ability to extract features [2], [5], [6], [7]. According to the research [5] of Jothimani, Shankar \& Surendra fluctuations between adjacent observations are better captured with Haar wavelet. Using discrete wavelet transformation in data processing, authors Chiang, Enke, Wu and Wang in their research [6] in the development of automated trading system achieved improved system performance by $87.80 \%$ from the previous approach that did not apply denoising. Significant improvements by using this technique are achieved by authors Jarrah and Salim [7], Their results show that the combination of discrete wavelet transformation and recurrent neural network allowed more accurate prediction of the day closing price versus the auto regressive integrated moving average (ARIMA).

Wavelet transformation is a promising, signal processing technique that simultaneously analyses the time domain and frequency domain. So, wavelet analysis allows us to extract hidden features from the original time series data or precisely, it can be used for filtering stock market data.

The paper investigates the impact of applying discrete wavelet transformation (DWT) in the data preparation phase on the quality of the learned model. Additionally, the accuracy of the model is compared with the results obtained for raw and misaligned data as well as for simple moving average (SMA) to show differences. In order to choose the best model for stock trend forecasting we use different artificial neural network architectures.

\section{Methodology}

In the preprocessing phase, in the first of the experiment combinations raw input financial time series data, while in the second and the third experiment combination simple moving average, as well as technique of discrete wavelet transformation are used.

In the second phase, the previously processed data are transmitted to one of the neural networks with the aim of predicting the trend of the stock price movement. The third phase measures the performance of the prediction model through the measure of performance accuracy. Below, the methods that were used for each of the phases are described in more details.

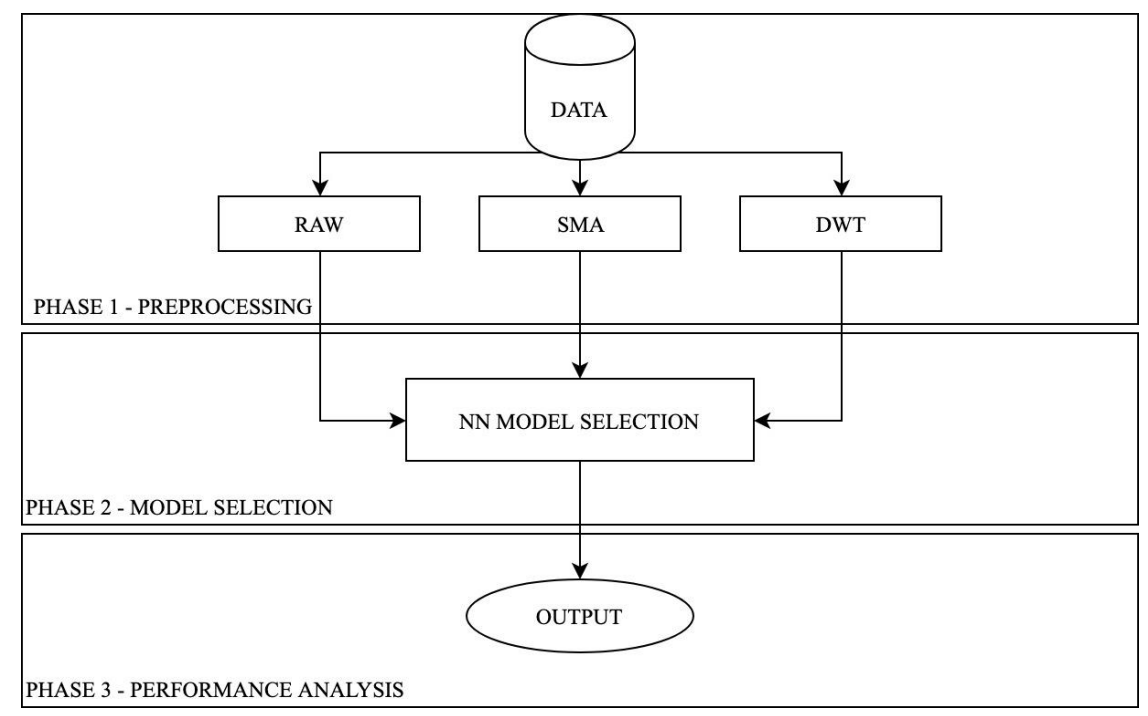

Fig. 1. Block diagram of methodology

\subsection{Preprocessing}

At this phase, as already mentioned, we use three combinations of input data shown on Fig. 1. The first combination is raw data without any preprocessing step. In the second combination we apply the simple moving average (SMA) (1) to all input variables.

$$
S M A=\frac{C_{t}+C_{t-1}+\cdots+C_{t-9}}{n}
$$


Discrete wavelet transformation (DWT) which was used in third combination is a mathematical model that allows the distribution of a given signal $O(t)$ to many frequency ranges. It finds its application in a number of areas, such as financial time series and signal processing, due to their strong ability to extract features [2], [7]. In the curse of the process of the signal decomposition through discrete wavelet transformation, the original signal $O(t)$ is divided into approximation coefficients $A(t)$ and $D(t)$. Therefore, component $A(t)$ contains a low frequency component of the signal $O(t)$ while component $D(t)$ contains high frequency components. In this research we have applied the Haar wavelet using Python library PyWavelets with nominal values to decompose the original signal $O(t)$. Its application is suitable for the analysis of time series as the method is able to catch fluctuations between adjacent observations [5].

\subsection{Model selection}

Feedforward neural network (FFNN) is often called in the literature a Multilayer Perceptrons (MLP) and it makes the foundation of the majority of deep learning models. These neural networks are mostly used to solve supervised learning tasks where we already know the target function as well as the result we want to achieve. The main goal of the feedforward neural network is to approximate a function $\mathrm{f}^{*}$. For example, the regression function $\mathrm{y}=\mathrm{f} *(\mathrm{x})$ maps the input of $\mathrm{x}$ to $\mathrm{y}$. The feedforward neural network defines the mapping of $y=f(x ; \theta)$ and also learns the value of the $\theta$ parameters which results in approximation of the best function [8].

When dealing with the feedforward neural network, data is moving only in one direction from the input layer to the output while at recurrent neural networks it can be said that data circles through the loop. Recurrent neural network (RNN) within each node uses a certain context unit that takes the information from the previous outputs and forwards that input information to the next layer. Because of this process, it is proved that recurrent neural network is extremely useful for sequential data that changes over time, because it will take into account previously learned information from the given time series [9].

There are some problems with the recurrent neural network when it comes to the calculation, and it is extremely difficult to successfully carry out its training process [9], so in order to address this problem a special long short-term memory (LSTM) cell has been developed [10]. Cells with long short-term memory have the ability to remove or add information to a particular cell regulated by the specific internal structures called gates [11]. The LSTM units have mechanisms for regulating the flow of information. The amount of input information that needs to be retained is systematically determined at each time step; therefore, LSTMs can memorize time patterns over a long period of time.

To implement these prediction models for the purpose of conducting the experiment, we used the Python programming language with the Tensorflow framework.

\subsection{Performance analysis}

Accuracy (ACC) is used as a measure of performance (2). Prediction accuracy is calculated as the ratio of the total number of correct predictions trend movement over the total of tested intervals.

$$
A C C=\frac{\text { correctly predicted }}{\text { total tested intervals }} \times 100
$$

\section{Experimental setup}

The experimental setup is closely related to the described methodology from the previous section in particular, with Fig 1. So, this section has three parts. In the first one, financial input data are described, processing data procedure are given in the second, and applied neural networks models are the subject of the last part.

\subsection{Data description}

We used the past financial time series data related to the value of selected stocks and stock index. These data include the value/price of the opening, the closing value/price, minimum value/price, maximum value/price and volume of trading, and the aforementioned were related to the shares of Microsoft, Facebook and the stock exchange index Dow Jones. The data was collected from a time period from January 1, 2010 to July 14, 2019 which makes up a total of about 2396 daily data sets. The data for this research are collected using the AlphaVantage API. 


\subsection{Data preprocessing}

Before prediction models all data were normalized within the limits of the -1 and 1 value to allow for a much faster training of models, the data were divided into a training set in a proportion of $80 \%$, and the rest of $20 \%$ data as the test set [12].

In this research, we measure the accuracy of the model by applying three different approaches to the processing of input data:

- The first approach involves the application of the unregulated raw data that we labelled as "RAW DATA".

- Within the second approach, we apply a ten-day simple moving average named "SMA".

- Within the third approach we use the discrete wavelet transformation technique - namely "DWT".

Graph in Fig. 2 shows the only one of input variables named closing price of the stock Microsoft where original price movement present raw data, de-noised price movement present discrete wavelet transformation technique and 10-day moving average price movement present simple moving average.
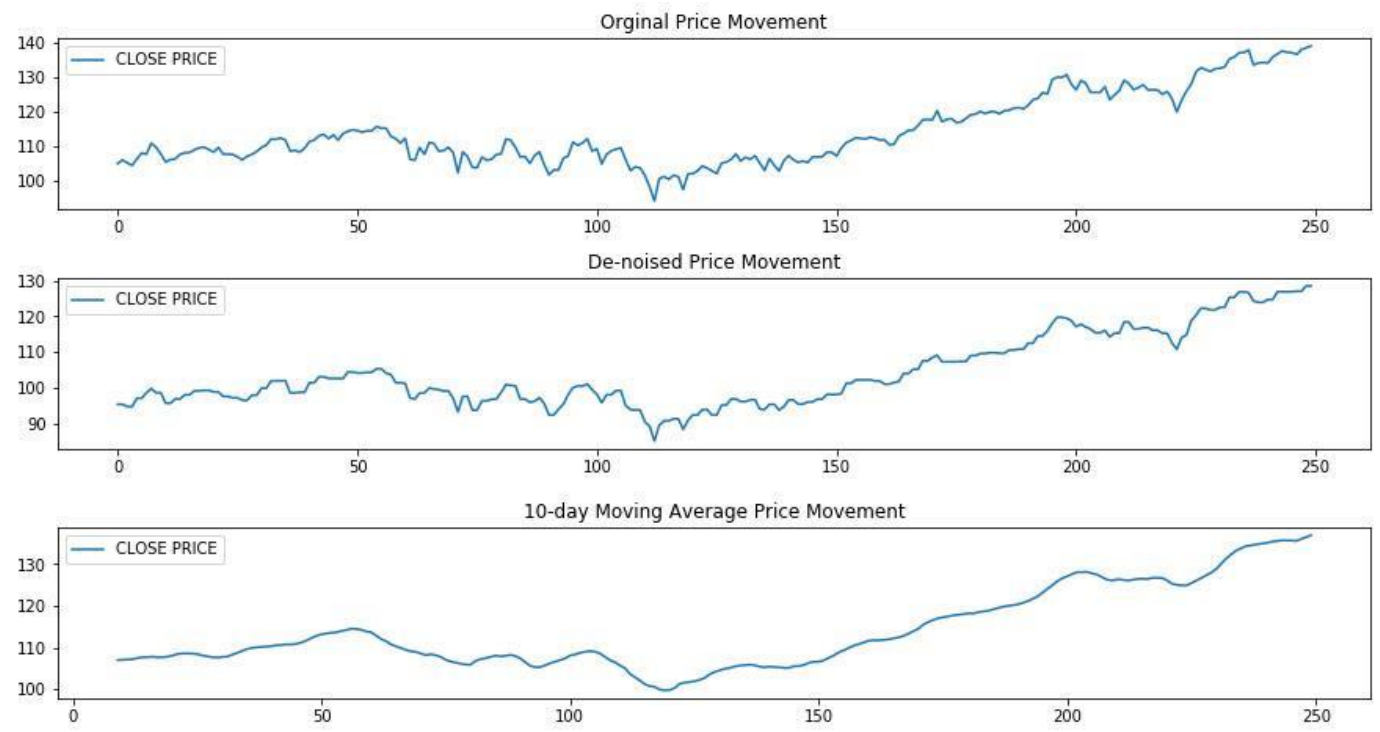

Fig. 2. Graph shows only close price with different preprocessing approaches.

\subsection{Prediction models}

In this paper we used three proposed prediction model based on neural networks: feedforward neural network (FFNN), recurrent neural network (RNN) and long short-term memory neural network (LSTM). Also, in the previous part of the section three types of input data series are presented where the goal is to show the results for each of them in order to see which data processing technique achieves the best performance results, in predicting of the future trend direction.

To ensure keeping the same conditions when training different models, the same hyper parameter settings, as well as the network architecture were used. These are the neural network that consists of five layers of which three hidden layers contain in line 256 neuronal structures in the first two layers, and 32 neurons in the last hidden layer. Furthermore, in all prediction models, Adaptive Moment Estimation (Adam) optimization algorithm and dropout techniques were used to ensure the exclusion of the network overfitting. To evaluate the performance of the model, we used the prediction accuracy indicator. It tells us how accurate the forecast model is at predicting the future trend movements.

\section{Results}

Results of prediction of the future trend direction on the stock market when using the applied model predictions and data processing techniques are shown in Table 1. From the table below, we can see that when it comes to the applied prediction models LSTM neural network generate better results than feedforward neural network and recurrent neural 
network. Also, when processing input data, we noticed a higher percentage of prediction accuracy when using discrete wavelet transformation than in the case where use the raw data or when applying a simple moving average.

\begin{tabular}{|c|c|c|c|c|}
\hline \multirow{2}{*}{ Company / Index } & \multirow{2}{*}{ Method } & \multicolumn{3}{|c|}{ Data Preprocessing Approaches } \\
\cline { 3 - 5 } & & RAW DATA & SMA & DWT \\
\hline \multirow{3}{*}{ Dow Jones (DJI) } & FFNN & $46.25 \%$ & $54.39 \%$ & $54.67 \%$ \\
\cline { 2 - 5 } & RNN & $46.76 \%$ & $53.46 \%$ & $54.58 \%$ \\
\cline { 2 - 5 } & LSTM & $47.60 \%$ & $53.04 \%$ & $\mathbf{5 5 . 1 1 \%}$ \\
\hline \multirow{3}{*}{ Microsoft (MSFT) } & FFNN & $45.29 \%$ & $41.19 \%$ & $42.29 \%$ \\
\cline { 2 - 5 } & RNN & $52.41 \%$ & $43.32 \%$ & $57.22 \%$ \\
\cline { 2 - 5 } & LSTM & $53.29 \%$ & $46.29 \%$ & $\mathbf{5 7 . 6 2 \%}$ \\
\hline \multirow{3}{*}{ Facebook (FB) } & FFNN & $51.11 \%$ & $46.93 \%$ & $49.17 \%$ \\
\cline { 2 - 5 } & RNN & $52.09 \%$ & $51.96 \%$ & $49.30 \%$ \\
\cline { 2 - 5 } & LSTM & $50.70 \%$ & $50.28 \%$ & $\mathbf{5 2 . 2 5 \%}$ \\
\hline
\end{tabular}

Table 1. Result of prediction model

From the results in Table 1. we can conclude that the use of DWT at the data preprocessing stage achieves better forecasts results, but also it should be noted that this is not the case in all experiments. In the case of Facebook stock, we can see that RNN leaves better results than LSTM, so we can conclude that the results do not depend only on the selected model but also depend on the selected stock.

\section{Conclusion and future research}

In this research, different neural network based models are applied in the task of forecasting trends on the stock market. The aim of the study is to compare the application of different neural network models in combination with different data processing techniques to achieve even better results.

With this research we have shown that using the discrete wavelet transformation for filtering the financial data time series we managed to achieve better results. With this technique, we can successfully remove certain noise from data and improve the quality of neural network model.

There's plenty of room for application and the improvement of prediction models in the stock market domain. One of the ideas for future research is the development of automated systems for trading on electronic stock exchanges using machine learning techniques. With the current achievements of selected forecasting models, numerous trading strategies can be implemented in the form of generating purchase and sales signals, in order to facilitate the decision-making process for marketers. Machine learning with application in the field of econometrics opens a completely new domain that has only just begun with its development.

\section{Acknowledgments}

This work has been fully supported by the University of Rijeka under the project number uniri-drustv-18-122.

\section{References}

[1] Hasan, N. \& Rasel, R. I. (2016). Artificial neural network approach for stock price and trend prediction, International conference on advanced information \& communication technology

[2] Chandar, S. K.; Sumathi, M. \& Sivanandam, S. N. (2016). Prediction of stock market price using hybrid of wavelet transform and artificial neural network. Indian Journal of Science and Technology, Vol. 9, No. 8 
[3] Liu, Y.; Qin, Z., Li, P. \& Wan, T. (2017). Stock volatility prediction using recurrent neural networks with sentiment analysis, In International Conference on Industrial, Engineering and Other Applications of Applied Intelligent Systems, pp. 192-201. Springer, Cham, DOI: 10.1007/978-3-319-60042-0_22

[4] Weng, B.; Ahmed, M. A. \& Megahed, F. M. (2017). Stock market one-day ahead movement prediction using disparate data sources, Expert Systems with Applications, Vol. 79, pp. 153-163, DOI: 10.1016/j.eswa.2017.02.041

[5] Jothimani, D.; Shankar, R. \& Yadav, S. S. (2016). Discrete wavelet transform-based prediction of stock index: a study on National Stock Exchange Fifty index, Journal of Financial Management and Analysis, Vol. 151, pp. 1017

[6] Chiang, W. C.; Enke, D.; Wu, T. \& Wang, R. (2016). An adaptive stock index trading decision support system, Expert Systems with Applications. Vol. 59, pp. 195-207, DOI: 10.1016/j.eswa.2016.04.025

[7] Jarrah, M. \& Salim, N. (2019). A recurrent neural network and a discrete wavelet transform to predict the Saudi stock price trends, International Journal of Advanced Computer Science and Applications. Vol. 10, No. 4, pp. 155162, DOI: 10.14569/IJACSA.2019.0100418

[8] Lecun, Y.; Bengio, Y. \& Hinton, H. (2015). Deep learning, Nature, Vol. 521, No. 7553, pp. 436-444

[9] Nielsen, M. A. (2015). Neural Networks and Deep Learning, Determination Press, Available from: http://neuralnetworksanddeeplearning.com

[10] Schmidhuber, J.; \& Hochreiter, S. (1997). Long short-term memory. Neural Computing, Vol. 9, No. 8, pp. 17351780, DOI: 10.1162/neco.1997.9.8.1735

[11] https://colah.github.io/posts/2015-08-Understanding-LSTMs/ (2015). Understanding LSTM networks. Accessed on: 2019-04-24

[12] Rechenthin, M. D. (2014). Machine-learning classification techniques for the analysis and prediction of highfrequency stock direction, Iowa Research Online, University of Iowa

[13] Trifan, A. L. (2011). Financial time series forecasting using neuro fuzzy approach for the Bucharest stock exchange, Annals of DAAAM for 2011 \& Proceedings of the 22nd International DAAAM Symposium, B. Katalinic (Ed.), Published by DAAAM International, ISSN 1726-9679, ISBN 978-3-901509-83-4 Vienna, Austria 